\title{
Gender differences in partners of patients with COPD and their perceptions about the patients
}

\author{
This article was published in the following Dove Press journal: \\ International journal of COPD \\ 23 December 2016 \\ Number of times this article has been viewed
}

\author{
Nienke Nakken' \\ Daisy JA Janssen ${ }^{1,2}$ \\ Monique van Vliet ${ }^{3}$ \\ Geeuwke J de Vries ${ }^{4}$ \\ Giny AL Clappers-Gielen ${ }^{5}$ \\ Arent Jan Michels ${ }^{6}$ \\ Jean WM Muris ${ }^{7}$ \\ Jan $\mathrm{H}$ Vercoulen ${ }^{8}$ \\ Emiel FM Wouters ${ }^{1,9}$ \\ Martijn A Spruit' \\ 'Department of Research and \\ Education, CIRO, Horn, ${ }^{2}$ Centre \\ of Expertise for Palliative Care, \\ Maastricht University Medical \\ Centre+ (MUMC+), Maastricht, \\ ${ }^{3}$ Department of Respiratory Medicine, \\ Zuyderland, Heerlen, ${ }^{4}$ Department \\ of Respiratory Medicine, Zuyderland, \\ Sittard-Geleen, ${ }^{5}$ Department of \\ Respiratory Medicine, Elkerliek \\ Hospital, Helmond, ${ }^{6}$ Department \\ of Respiratory Medicine, St Anna \\ Hospital, Geldrop, ${ }^{7}$ Department of \\ Family Medicine, CAPHRI School \\ of Public Health and Primary Care, \\ Maastricht University, Maastricht, \\ ${ }^{8}$ Department of Medical Psychology \\ and Department of Pulmonary \\ Diseases, Radboud University \\ Nijmegen Medical Centre, Nijmegen, \\ 'Department of Respiratory Medicine, \\ Maastricht University Medical \\ Centre+ (MUMC+), Maastricht, \\ the Netherlands
}

Background/objectives: Chronic obstructive pulmonary disease (COPD) not only affects patients but also their partners. Gender-related differences in patients with COPD are known, for instance regarding symptoms and quality of life. Yet, research regarding gender differences in partners of patients with COPD has been conducted to a lesser extent, and most research focused on female partners. We aimed to investigate differences between male and female partners of patients with COPD regarding their own characteristics and their perceptions of patients' characteristics.

Design: Cross-sectional study.

Setting: Four hospitals in the Netherlands.

Participants: One hundred and eighty-eight patient-partner couples were included in this cross-sectional study.

Measurements: General and clinical characteristics, health status, care dependency, symptoms of anxiety and depression, social support, caregiver burden, and coping styles were assessed during a home visit.

Results: Female partners had more symptoms of anxiety and a worse health status than male partners. Social support and caregiver burden were comparable, but coping styles differed between male and female partners. Female partners thought that male patients were less care dependent and had more symptoms of depression, while these gender differences did not exist in patients themselves.

Conclusion: Health care providers should pay attention to the needs of all partners of patients with COPD, but female partners in particular. Obtaining an extensive overview of the patientpartner couple, including coping styles, health status, symptoms of anxiety, and caregiver burden, is necessary to be able to support the couple as effectively as possible.

Keywords: COPD, family caregiving, gender, partners

\section{Introduction}

Chronic obstructive pulmonary disease (COPD) is a preventable and treatable disease, characterized by a usually progressive, persistent airflow limitation. ${ }^{1}$ Although once seen as a disease of men, nowadays COPD is known to be increasingly prevalent among women. ${ }^{2}$ As COPD becomes more prevalent in women, several studies have focused on possible gender-related differences. For instance, women with COPD report more symptoms such as dyspnea, fatigue, chronic cough, anxiety, and depression, but they also report a lower health-related quality of life compared to their male peers. ${ }^{3-6}$

Patients' symptom burden can result into problems with activities of daily life, which, in turn can result in care dependence on family members and in particular partners. ${ }^{7}$ Yet, research regarding possible gender differences in partners of patients with COPD has been conducted to a lesser extent, and most research focused on female partners. ${ }^{8,9}$ Indeed, compared to male family caregivers, female family caregivers
Correspondence: Nienke Nakken Department of Research and Education, CIRO, Hornerheide I, 6085 NM Horn, the Netherlands

Tel +3l 475587602

Email nienkenakken@ciro-horn.nl
International Journal of COPD 2017:12 95-104

95

Dovepress

http://dx.doi.org// 0.21 477/COPD.SI I887| (c) (1) (5) 2017 Nakken et al. This work is published and licensed by Dove Medical Press Limited. The full terms of this license are available at https://www.dovepress.com/terms.php cc) hereby accept the Terms. Non-commercial uses of the work are permitted without any further permission from Dove Medical Press Limited, provided the work is properly attributed. For permission for commercial use of this work, please see paragraphs 4.2 and 5 of our Terms (https://www.dovepress.com/terms.php). 
of patients with COPD reported a higher prevalence of increased symptoms of anxiety and depression. ${ }^{10}$ This is in line with findings in female family caregivers of patients from the general population, hospice patients, and patients with cancer. ${ }^{11-13}$ These female caregivers, compared to male caregivers, have more difficulty with balancing informal caregiving with other (family and employment) responsibilities, suffer from poorer emotional health, ${ }^{11}$ and appear to be more distressed. ${ }^{12}$ Moreover, they show a more negative impact on their daily activities, health, and family support ${ }^{13}$ compared to men. It seems that males' distress is related to their own health condition and not to the health condition of their female patient partner, while this is different in women. ${ }^{14}$ Another explanation may be that female informal caregivers use different coping strategies, such as seeking social support and wishful thinking, as was shown in caregivers of patients with dementia. ${ }^{15}$ Partners' perceptions of the patients' health status may also explain differences in the experience of caregiving. Indeed, a previous study in patients with COPD, chronic heart failure, or chronic renal failure showed major differences between the patients' symptom report and their family caregivers' perception. ${ }^{16}$ It is reasonable to assume that gender differences do exist in partners' perceptions of patients' health status, care dependency, and symptoms of anxiety and depression. These gender differences in partners' perceptions may also explain gender differences in the experience of caregiving. To date, it remains unknown whether gender differences in partners' perceptions of patients with COPD actually do exist.

Therefore, we aimed to investigate differences between male and female partners of patients with COPD not only regarding their own health status, symptoms of anxiety and depression, social support, caregiver burden, and coping styles but also regarding their perceptions of the patients' care dependency, symptoms of anxiety and depression, and health status. We hypothesized that female partners have a worse health status, more symptoms of anxiety and depression, and perceive lower levels of social support and higher levels of caregiver burden themselves. Moreover, we hypothesized that coping styles such as seeking social support and reassuring thoughts are more important for female than for male partners. Additionally, we hypothesized that female partners perceive a worse health status, more symptoms of anxiety and depression, and more care dependency in patients compared to male partners.

\section{Methods}

\section{Design}

The baseline results of the Home Sweet Home Study were used, in which data were collected during home visits at baseline and after 12 months. ${ }^{17}$ Additional home visits were performed when an exacerbation-related hospital admission occurred during the 12-month follow-up period. This study is approved by the Medical Research Ethics Committees United, the Netherlands (NL42721.060.12/M12-1280), and is registered in the Dutch Trial Register (NTR3941). The protocol of this study and data about health status and problematic activities of daily life were published before. ${ }^{17-19}$

\section{Study population}

Patients with COPD were recruited by their chest physician or a respiratory nurse specialist in four hospitals throughout the southern-eastern part of the Netherlands. Additionally, patients participating in the "Chance study" (NTR3416) ${ }^{20}$ who met the inclusion criteria of the Home Sweet Home study and were willing to participate in further research were asked to participate in the current study. Inclusion occurred during the first home visit, between July 2013 and December 2014.

Patients were eligible if they had moderate to very severe COPD (Global initiative for chronic Obstructive Lung Disease [GOLD] grade II, III, or IV); ${ }^{21}$ no exacerbation of $\mathrm{COPD}^{22}$ or hospitalization $<4$ weeks preceding enrolment; and if they could identify a loved one (defined as a person living together with the patient with COPD, regardless of whether or not he/she provides [informal] care to the patient with COPD). Patients and/or partners were excluded if they were unable to complete the study questionnaires because of cognitive impairment (defined as Short Blessed Test score $\geq 10$ point $)^{23}$ or if they were unable to understand Dutch. Furthermore, non-partner relationships and nonheterosexual patient-partner pairs were excluded in the current analysis. All participating patients and their participating partners gave written informed consent.

\section{Instruments}

Demographics and clinical characteristics were assessed in both patients and partners, during a visit in their home environment. This included body mass index (weight/ height $^{2}$ ), self-reported comorbidities (Charlson comorbidity index), ${ }^{24}$ smoking history and current smoking habits, and post-bronchodilator spirometry (to calculate the forced expiratory volume in the first second and Tiffeneau index, using a handheld spirometer according to guidelines). ${ }^{1}$ In patients, the total number of COPD exacerbations with and without hospital admission in the year before the home visit, and receiving help with personal care from professionals or relatives during the last 6 months before the home visit were assessed. 
The Dutch relationship questionnaire (Nederlandse Relatievragenlijst) was used to assess the quality of the relationship between the patient and partner. ${ }^{25}$ This selfadministered questionnaire was assessed in both patients and partners separately and consists of 80 questions in five domains (independence, emotional solidarity, identity, conflict handling, and sexuality). Its total score ranges from 0 (very low) to 80 points (very high quality of the relationship).

Care dependency was assessed using the care dependency scale, which consists of 15 items. ${ }^{26}$ The total score ranges from 15 (worst) to 75 points (best), whereby patients with a score $\leq 68$ points were considered as care dependent. ${ }^{27}$ This questionnaire was assessed in patients, partners, and partners' perception of the patients.

Coping styles, ie, the way a person (partner) deals with problems or stressful situations, were assessed using the Utrecht Coping List. ${ }^{28}$ It consists of 47 items divided into seven subscales:

1. Active confronting: confronting problems and employing purposeful strategies. Item example: "Immediately intervene if there are difficulties."

2. Palliative reaction: distracting one's attention from the problems, includes smoking and drinking. Item example: "Trying to relax."

3. Avoidance: waiting and keeping clear of the problem. Item example: "Avoid difficult situations."

4. Seeking social support: seeking comfort and help from others. Item example: "Sharing your concerns with someone."

5. Passive reaction pattern: worrying and drawing back. Item example: "Isolate yourself from others."

6. Expressing emotions: showing annoyance or anger. Item example: "Release your tension."

7. Reassuring thoughts: self-encouragement and realizing that worse things can happen. Item example: "Tell yourself that it will turn out better than expected."

Each item has four levels: seldom or never, sometimes, often, and very often. A higher score indicates a greater amount of the variable being measured.

Symptoms of anxiety and depression in patients, partners, and partners' perceptions of patients were assessed using the Hospital Anxiety and Depression Scale (HADS) ${ }^{29}$ This scale is divided into an anxiety subscale (HADS-A) and a depression subscale (HADS-D). Total scores, for both subscales, range from 0 (optimal) to 21 points (worst). In addition, a score $\geq 10$ points for each subscale was considered as a clinically relevant symptom of anxiety or depression.

Generic health status was assessed using the EuroQol-5 Dimensions (EQ-5D) and the Assessment of Quality of Life with 8 Dimensions (AQoL-8D). The EQ-5D included five questions about health-related quality of life and provided an index score (Dutch version) between -0.33 (worst) and 1.0 (best). In addition, current health was rated on a visual analogue scale, which ranges from 0 (worst possible health) to 100 (best possible health). The AQoL-8D is more comprehensive with 35 questions in eight dimensions of healthrelated quality of life. These eight dimensions can also be combined into a mental and physical health dimension and a utility score. All scales range from 0.00 (death) to 1.00 (best health). The EQ-5D and AQoL-8D were assessed in patients and partners themselves, and in partners' perceptions of the patients.

Social support perceived by patients and partners themselves was assessed using the Medical Outcome Study Social Support Survey. ${ }^{30}$ This multidimensional questionnaire contained 19 items regarding five dimensions of social support: emotional support, informational support, tangible support, positive social interaction, and affectionate support. Each item has five response choices to indicate how often support was available to them if they needed it, namely: 1) none of the time, 2) a little of the time, 3) some of the time, 4) most of the time, and 5) all of the time. A higher score indicates a greater amount of that specific kind of support. Additionally, the total number of close friends and relatives was asked.

To assess caregiver burden in resident partners, the Family Appraisal of Caregiving Questionnaire for Palliative Care was used. ${ }^{31}$ This 25 -item questionnaire includes caregiver burden and positive aspects of caregiving in four domains: caregiver strain, positive caregiving appraisals, caregiver distress, and family well-being. The item scores range from 1 (strongly disagree) to 5 points (strongly agree), so a higher score means a greater amount of the variable being measured.

\section{Statistics}

Categorical variables are described as frequencies, while continuous variables were tested for normality and are presented as mean and standard deviation (SD). Continuous variables were compared between male and female partners using independent samples $t$-tests or Mann-Whitney $U$-tests, as appropriate. Categorical variables were compared between male and female partners using the chi-square tests. In a post hoc analysis, we compared coping styles in female partners with and without symptoms of anxiety. Moreover, we checked for differences in patients' health status between patients of partners with and without symptoms of depression. Post hoc Pearson's product-moment correlations or Spearman's rank correlations, depending on the variable 
distribution, were performed to measure the association between caregiver burden and partners' social support. Strength of the correlation was classified as: no relationship $(<0.25)$, fair (0.25-0.50), moderate to good (0.50-0.75), and good to excellent $(>0.75) .{ }^{32}$ A priori, the level of significance was set at $P \leq 0.01$, because of multiple comparisons. All statistics were performed using SPSS version 20.0 (IBM, lnc., Chicago, IL, USA) or GraphPad Prism 6 (GraphPad Software, La Jolla, CA, USA).

\section{Results}

\section{General characteristics}

In total, 569 patients were screened for eligibility, 449 patient-partner pairs met the inclusion criteria, and 194 pairs completed the home visit. Six pairs were excluded for this analysis due to non-partner relationships or non-heterosexual relationships (Supplementary materials). Therefore, 188 pairs ( $42 \%$ of eligible patient-partner pairs) were included. No differences were found between participants and eligible patients refusing participation regarding age, gender distribution, and GOLD grade (all $P>0.05$ ). In total, 85 partners were males $(45.2 \%)$ and 103 were females $(54.8 \%)$. On average, partners and patients had similar age (65.3 [8.7] versus 66.3 [8.6] years, respectively, $P=0.274$ ). Most patients had COPD GOLD II (47.9\%), followed by COPD GOLD III (32.4\%) and COPD GOLD IV (19.7\%). The majority of the patients had a modified Medical Research Council scale dyspnea score of $\geq 2$ (77.7\%). Moreover, $16.5 \%$ of the patients and $32.4 \%$ of the partners were current smokers.

\section{Male versus female patients}

Female patients were significantly younger than male patients and had better EQ-5D index scores (Table 1). Other general and clinical characteristics, care dependency, and symptoms of anxiety and depression were comparable between male and female patients (Table 1).

\section{Male versus female partners}

Male and female partners were comparable regarding general and clinical characteristics, except for higher number of smoking pack years in male partners (Table 2).

Table I Patient characteristics, symptoms of anxiety and depression, and health status

\begin{tabular}{|c|c|c|c|}
\hline & $\begin{array}{l}\text { Female patients } \\
(n=85)\end{array}$ & $\begin{array}{l}\text { Male patients } \\
(n=103)\end{array}$ & $P$-value \\
\hline \multicolumn{4}{|l|}{ General and clinical characteristics } \\
\hline Age, years & $63.3(8.0)$ & $68.7(8.3)$ & $<0.001$ \\
\hline $\mathrm{BMI}, \mathrm{kg} / \mathrm{m}^{2}$ & $25.8(5.4)$ & $26.8(5.2)$ & 0.240 \\
\hline Charlson comorbidity index (patients) ${ }^{\mathrm{a}}$ & $2.2(1.3)$ & $2.5(1.6)$ & 0.141 \\
\hline Current smoker & 19 (22.4\%) & $12(11.7 \%)$ & 0.049 \\
\hline Pack years ${ }^{\mathrm{a}}$ & $36.4(17.5)$ & $43.3(21.8)$ & 0.068 \\
\hline $\mathrm{FEV}_{1}(\%$ predicted $)$ & $47.7(18.0)$ & $46.7(17.5)$ & 0.697 \\
\hline \multicolumn{4}{|l|}{ Number of exacerbations in previous year } \\
\hline Without hospital admission ${ }^{\mathrm{a}}$ & $1.64(1.73)$ & $\mathrm{I} .8 \mathrm{I}(2.02)^{\mathrm{b}}$ & 0.718 \\
\hline With hospital admission ${ }^{\mathrm{a}}$ & $0.73(1.34)$ & $0.90(1.30)$ & 0.186 \\
\hline Receiving care in past 6 months & & & 0.375 \\
\hline From professionals & 19 (22.4\%) & $19(18.4 \%)$ & \\
\hline From relatives & $11(12.9 \%)$ & $21(20.4 \%)$ & \\
\hline Dutch relationship questionnaire (patients) ${ }^{\mathrm{a}}$ & $62.5(13.9)^{c}$ & $60.4(13.4)^{d}$ & 0.115 \\
\hline Care dependency scale (patients) ${ }^{\mathrm{a}}$ & $68.9(7.6)$ & $67.1(9.5)^{b}$ & 0.294 \\
\hline \multicolumn{4}{|l|}{ Anxiety, depression, and health status } \\
\hline Anxiety subscale (HADS-A) & $5.7(3.5)$ & $5.8(3.6)$ & 0.940 \\
\hline Depression subscale (HADS-D) & $5.2(3.3)$ & $6.1(3.9)$ & 0.072 \\
\hline Symptoms of anxiety $(\geq 10), \mathrm{n}(\%)$ & $13(15.3)$ & $15(14.6)$ & 0.889 \\
\hline Symptoms of depression ( $\geq 10), n(\%)$ & II (I2.9) & $18(17.5)$ & 0.392 \\
\hline EQ-5D index score & $0.76(0.22)$ & $0.68(0.27)$ & 0.010 \\
\hline EQ-5D VAS score & $64.8(17.2)$ & $60.0(19.9)$ & 0.081 \\
\hline AQoL-8D, mental & $0.34(0.16)$ & $0.35(0.18)$ & 0.754 \\
\hline AQoL-8D, physical & $0.53(0.18)$ & $0.55(0.17)$ & 0.540 \\
\hline AQoL-8D, utility score & $0.63(0.18)$ & $0.64(0.18)$ & 0.821 \\
\hline
\end{tabular}

Notes: Values expressed as mean (SD) or number of patients (\%). ${ }^{a}$ Non-parametric statistical tests have been used because of skewed data; ${ }^{b} \mathrm{l}=\mathrm{l}$ missing; ${ }^{\mathrm{n}} \mathrm{n}=5 \mathrm{missing}$; ${ }^{\mathrm{d}} \mathrm{n}=9$ missing.

Abbreviations: AQoL-8D, Assessment of Quality of Life with 8 Dimensions; BMI, body mass index; EQ-5D, EuroQol-5 dimensions; FEV , forced expiratory volume in the first second; HADS-A, Hospital Anxiety and Depression Scale, Anxiety subscale; HADS-D, Hospital Anxiety and Depression Scale, Depression subscale; SD, standard deviation; VAS, visual analog scale. 
Table 2 Male versus female partners

\begin{tabular}{|c|c|c|c|}
\hline & $\begin{array}{l}\text { Male partners } \\
(n=85)\end{array}$ & $\begin{array}{l}\text { Female partners } \\
(n=103)\end{array}$ & $P$-value \\
\hline \multicolumn{4}{|l|}{ General and clinical characteristics } \\
\hline Age, years & $65.1(8.9)$ & $65.4(8.6)$ & 0.808 \\
\hline $\mathrm{BMI}, \mathrm{kg} / \mathrm{m}^{2}$ & $28.2(4.6)^{b}$ & $27.7(5.1)^{b}$ & 0.227 \\
\hline Charlson comorbidity index (patients) & $1.4(1.7)$ & I.I (I.5) & 0.449 \\
\hline Current smoker & $29(34.1 \%)$ & $32(31.1 \%)$ & 0.657 \\
\hline Pack years ${ }^{\mathrm{a}}$ & $26.7(21.7)$ & $16.3(18.5)$ & $<0.001$ \\
\hline Tiffeneau index $<70 \%$, n (\%) & $29(34.1)^{b}$ & $27(26.2)^{c}$ & 0.269 \\
\hline $\mathrm{FEV}_{1}(\% \text { predicted })^{\mathrm{a}}$ & $100.4(27.6)^{b}$ & $107.1(23.7)^{c}$ & 0.066 \\
\hline Dutch relationship questionnaire (patients) & $62.7(11.3)^{d}$ & $58.8(14.6)^{\mathrm{e}}$ & 0.053 \\
\hline Care dependency scale (patients) ${ }^{\mathrm{a}}$ & $74.2(2.1)$ & $73.4(6.4)^{b}$ & 0.946 \\
\hline \multicolumn{4}{|l|}{ Anxiety, depression and health status } \\
\hline Anxiety subscale (HADS-A) & $4.5(3.3)$ & $6.9(4.1)$ & $<0.001$ \\
\hline Depression subscale (HADS-D) ${ }^{\mathrm{a}}$ & $3.7(2.5)$ & $4.3(3.1)$ & 0.261 \\
\hline Symptoms of anxiety ( $\geq 10), \mathrm{n}(\%)$ & $7(8.2)$ & $31(30.1)$ & $<0.001$ \\
\hline Symptoms of depression $(\geq 10), n(\%)$ & $3(3.5)$ & $4(3.9)$ & 0.898 \\
\hline EQ-5D, index score ${ }^{\mathrm{a}}$ & $0.93(0.1 \mathrm{I})^{\mathrm{b}}$ & $0.86(0.17)$ & 0.001 \\
\hline EQ-5D, VAS score ${ }^{\mathrm{a}}$ & $83.5(\mid 4.4)^{b}$ & $81.9(14.2)$ & $0.4 \mid 4$ \\
\hline AQoL-8D, mental & $0.52(0.16)$ & $0.43(0.15)^{b}$ & $<0.001$ \\
\hline AQoL-8D, physical & $0.74(0.18)$ & $0.69(0.21)^{b}$ & 0.109 \\
\hline AQoL-8D, utility score ${ }^{a}$ & $0.82(0.14)$ & $0.75(0.14)^{b}$ & $<0.001$ \\
\hline \multicolumn{4}{|l|}{ Social support } \\
\hline Good friends or close relatives ${ }^{a}$ & II (I3) & $12(10)^{b}$ & 0.368 \\
\hline Emotional/informational support & $3.9(0.9)$ & $4.1(0.9)^{c}$ & 0.222 \\
\hline Tangible support & $4.0(0.9)$ & $4.0(1.0)^{f}$ & 0.897 \\
\hline Affectionate support $\mathrm{t}^{\mathrm{a}}$ & $4.3(0.8)$ & $4.3(0.8)^{c}$ & 0.521 \\
\hline Positive social interaction & $4.0(0.9)^{b}$ & $4.2(0.8)^{f}$ & 0.245 \\
\hline Overall support index & $4.0(0.8)^{b}$ & $4.1(0.8)^{d}$ & 0.387 \\
\hline \multicolumn{4}{|l|}{ Caregiver burden } \\
\hline Caregiver strain & I.5 (0.7) & $\mathrm{I} .6(0.7)$ & 0.528 \\
\hline Positive caregiving appraisals & $3.9(1.2)$ & $3.8(1.2)$ & 0.401 \\
\hline Caregiver distress & $2.0(1.0)$ & $2.1(1.0)$ & 0.724 \\
\hline Family well-being ${ }^{a}$ & $3.9(0.7)$ & $3.7(0.9)$ & 0.452 \\
\hline
\end{tabular}

Notes: Values expressed as mean (SD) or number of partners (\%). ${ }^{a}$ Non-parametric statistical tests have been used because of skewed data; ${ }^{b} n=I$ missing; ${ }^{c} n=3$ missing; ${ }^{d} n=4$ missing; ${ }^{e} n=7$ missing; ${ }^{f} n=2$ missing.

Abbreviations: AQoL-8D, Assessment of Quality of Life with 8 Dimensions; BMI, body mass index; EQ-5D, EuroQol-5 dimensions; FEV , forced expiratory volume in the first second; HADS-A, Hospital Anxiety and Depression Scale, Anxiety subscale; HADS-D, Hospital Anxiety and Depression Scale, Depression subscale; VAS, visual analog scale; SD, standard deviation.

Six out of the total seven subscales of the questionnaire about coping showed significantly different outcomes for male partners compared to female partners (Figure 1). Very low levels of active confronting, very high levels of palliative reaction, and high levels of avoidance were found significantly more often in female partners compared to male partners. Low levels of seeking social support were more often found in female partners, while medium levels were more common in male partners. In contrast, (very) low levels of the subscales passive reaction pattern and reassuring thoughts were significantly more often found in male partners, while high levels were more common in female partners.

Post hoc analysis showed that low levels of active confronting and high levels of passive reaction pattern were found significantly more often in female partners with symptoms of anxiety compared to female partners without these symptoms (Supplementary materials). In addition, medium levels of passive reaction pattern were found significantly more often in female partners without anxiety symptoms compared to female partners with symptoms of anxiety. Moreover, female partners had worse mean HADS-A scores, EQ-5D index scores, AQoL-8D mental health dimension scores, and AQoL-8D utility scores (Table 2). HADS-D scores, social support, and caregiver burden were comparable between male and female partners. Post hoc analysis showed that patients of a partner with clinically relevant symptoms of depression scored significantly worse on the AQoL-8D physical and utility scores (Supplementary materials). Furthermore, the caregiver strain subscale showed a fair and significant relationship with the Medical Outcome Study Social Support Survey subscales "emotional/informational 


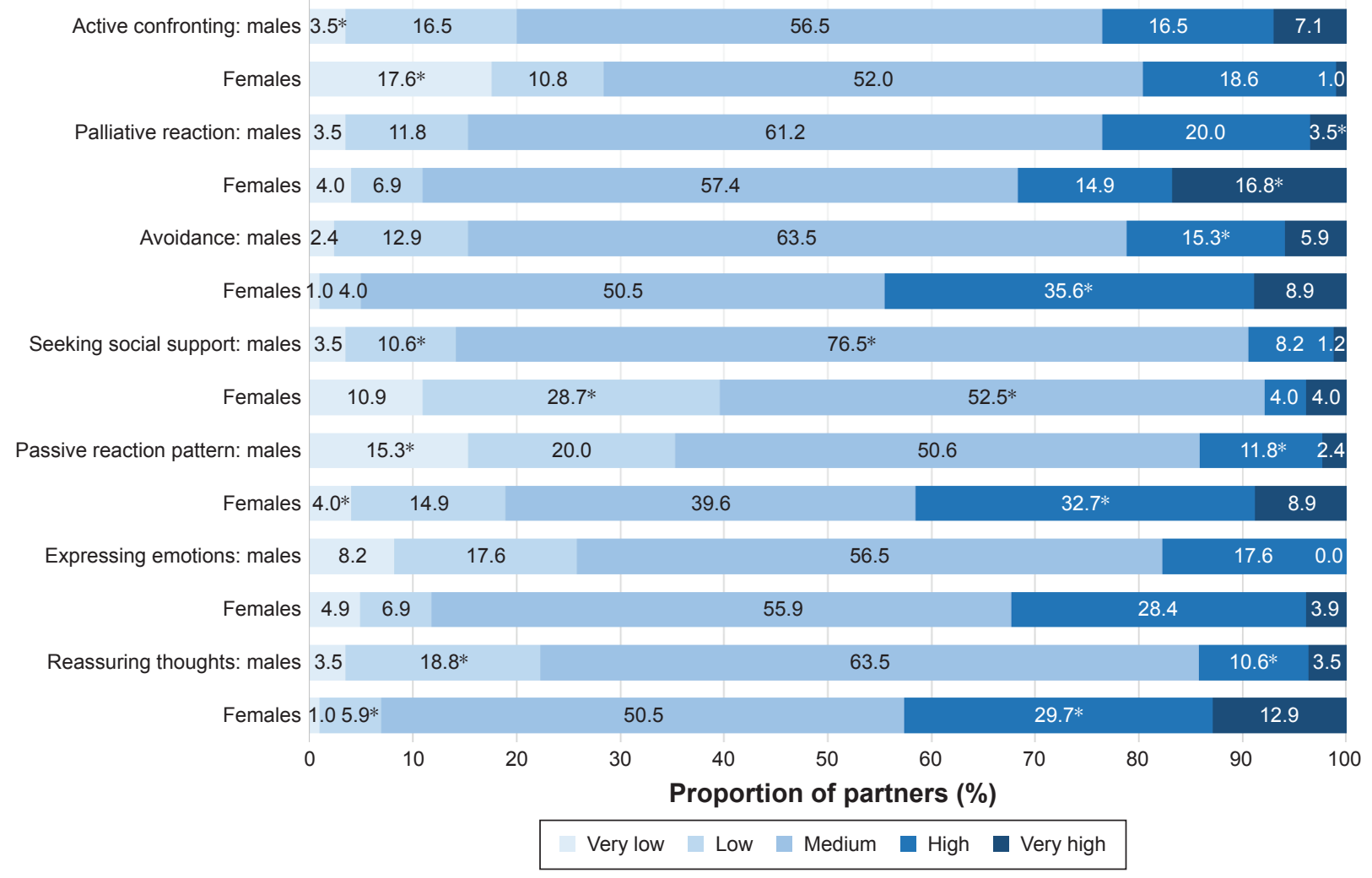

Figure I Categorical Utrecht Coping List scores in partners, after stratification for gender.

Notes: Proportion of partners using a very low, low, medium, high, or very high level of a specific coping style. $* P \leq 0.0$ I, compared with the same category of the same subcale for the other gender.

support," "tangible support," and the "overall support index" (Supplementary materials). No relationship was found for the caregiver distress subscale (Supplementary materials).

\section{Partners' perceptions of patients' care dependency, mood, and health status}

Male partners gave female patients a significantly lower score on the care dependency scale compared to female partners about male patients (Table 3). However, no differences were found between male and female partners' perceptions of the proportion of care-dependent patients (32.9\% vs $24.3 \%$, respectively, $P=0.189$ ) (Supplementary materials).

Moreover, female partners gave their male patients a higher score on the HADS depression subscale. Although an equal amount of male and female patients $(P=0.392$, Table 1) reported symptoms of depression (shown in the

Table 3 Partners' perceptions of patients' care dependency, symptoms of anxiety and depression, and health status

\begin{tabular}{|c|c|c|c|}
\hline & $\begin{array}{l}\text { Male partners } \\
(n=85)\end{array}$ & $\begin{array}{l}\text { Female partners } \\
(n=103)\end{array}$ & P-value \\
\hline \multicolumn{4}{|l|}{ Care dependency } \\
\hline Care dependency scale (patients) ${ }^{\mathrm{a}}$ & $68.2(9.0)$ & $70.4(7.9)$ & 0.003 \\
\hline \multicolumn{4}{|l|}{ Anxiety, depression, and health status } \\
\hline Anxiety subscale (HADS-A) & $6.1(3.7)^{\mathrm{b}}$ & $6.0(3.8)$ & 0.915 \\
\hline Depression subscale (HADS-D) & $4.7(3.0)^{\mathrm{b}}$ & $6.3(3.7)$ & 0.001 \\
\hline Symptoms of anxiety $(\geq 10), n(\%)$ & $15(17.6)^{b}$ & $22(21.4)$ & 0.550 \\
\hline Symptoms of depression $(\geq 10), n(\%)$ & $6(7.1)^{b}$ & $22(21.4)$ & 0.007 \\
\hline EQ-5D index score & $0.74(0.23)^{\mathrm{b}}$ & $0.68(0.29)$ & 0.082 \\
\hline EQ-5D VAS score & $64.1(17.8)^{b}$ & $61.1(21.8)$ & 0.299 \\
\hline AQoL-8D, mental & $0.38(0.18)^{b}$ & $0.35(0.18)$ & 0.221 \\
\hline AQoL-8D, physical & $0.51(0.18)^{b}$ & $0.52(0.18)$ & 0.784 \\
\hline AQoL-8D, utility score & $0.65(0.20)^{\mathrm{b}}$ & $0.62(0.20)$ & 0.359 \\
\hline
\end{tabular}

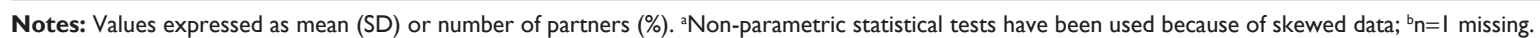

Abbreviations: AQoL-8D, Assessment of Quality of Life with 8 Dimensions; EQ-5D, EuroQol-5 dimensions; HADS-A, Hospital Anxiety and Depression Scale, Anxiety subscale; HADS-D, Hospital Anxiety and Depression Scale, Depression subscale; VAS, visual analog scale; SD, standard deviation. 


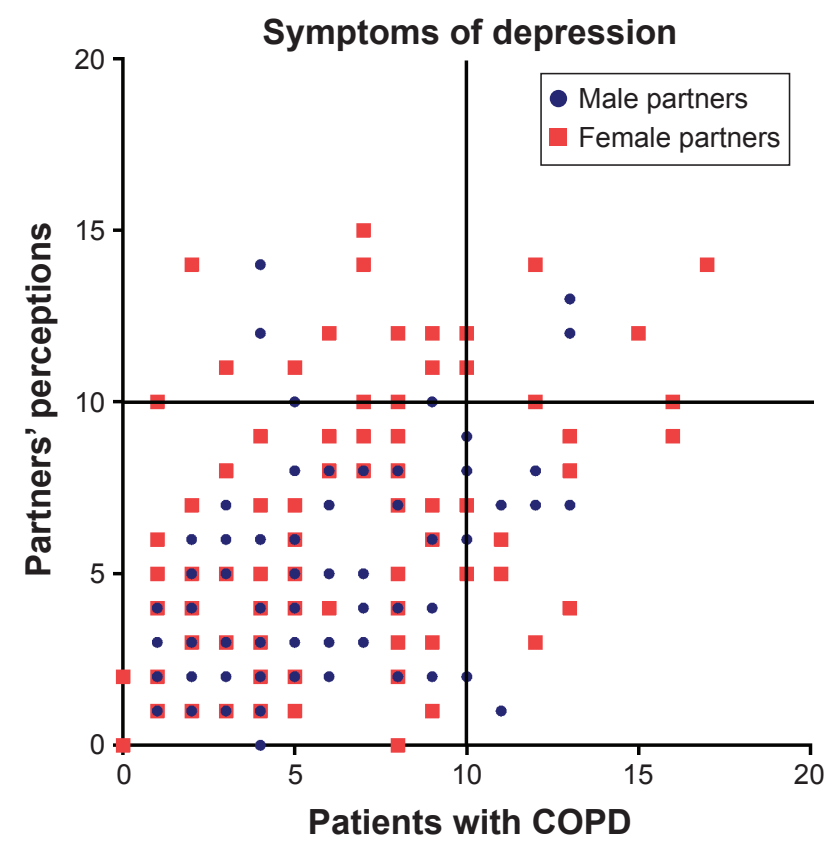

Figure 2 Patients' and partners' perceptions about patients' symptoms of depression Note: HADS-D scores of patients with COPD (horizontal axes) and partners' perceptions of the patients (vertical axes).

Abbreviations: COPD, chronic obstructive pulmonary disease; HADS-D, Hospital Anxiety and Depression Scale, Depression subscale.

two right quadrants in Figure 2), more female partners than male partners perceived clinically relevant symptoms of depression in patients $(P=0.007$, Table 3$)$ (shown in the two upper quadrants in Figure 2). In addition, a significant difference between male and female patients was found for the EQ-5D index score (Table 1). However, male and female partners scored the patients not significantly different on the EQ-5D (Table 3).

\section{Discussion Key findings}

Female partners had more symptoms of anxiety and a worse health status than male partners, but social support and caregiver burden were comparable for male and female partners of patients with COPD. Coping styles differed between male and female partners. Moreover, female partners thought that male patients were less care dependent and had more symptoms of depression, while these gender differences did not exist in patients themselves.

\section{Male versus female partners}

This study showed that female partners have more often clinically relevant symptoms of anxiety compared to male partners, which is in line with a study by Jácome et al. ${ }^{10}$ Factors which might contribute to the higher levels of anxiety in women are as follows: women might be more emotionally attentive and more likely to report negative emotions, ${ }^{33,34}$ they use more emotion-focused coping styles, ${ }^{33}$ and perform more time-consuming caregiving tasks and household chores. ${ }^{35}$ In the current study, equal levels of depression in men and women were found. These findings are in line with a large population-based study providing normative data of the United Kingdom. ${ }^{36}$ Depressive symptoms in caregivers of patients with chronic heart failure and terminally ill cancer patients were related to patients' disease burden..$^{37,38}$ Therefore, the current authors conducted a post hoc analysis, which showed that patients had a worse health status when their partner had symptoms of depression.

Comparable with other studies, ${ }^{39,40}$ we found no differences in caregiver burden between male and female partners. Moreover, we did not find any difference in the quality of the relationship and levels of social support between male and female patients and partners. The equal levels of burden found in this study might be explained by the equal levels of social support in partners, because more caregiver burden could be experienced when perceiving less social support. ${ }^{41}$ By conducting post hoc correlations, we confirmed that lower levels of social support were associated with higher levels of caregiver strain.

This study showed differences in categorical subscales of coping between male and female partners, which was also found in a random sample of the Swedish population. ${ }^{42}$ These gender differences could be related to the higher anxiety scores in female partners. In fact, female partners in the current study reported similar levels of coping compared to patients with symptoms of anxiety in a previous study, ${ }^{43}$ namely, a lower use of active confronting style and an increased use of palliative reaction, avoidance, and passive reaction pattern. Therefore, we conducted a post hoc analysis in female partners of patients with COPD. The post hoc results were in line with the results of Stoilkova et al. ${ }^{43}$

\section{Partners' perceptions of patients' care dependency, symptoms of anxiety and depression, and health status}

Male partners thought that female patients were more care dependent than female partners did, while male and female patients' scores were comparable. No differences were found between male and female partners' perceptions regarding patients' health status. However, a small difference on the EQ-5D index score did exist between male and female patients themselves. So it seems reasonable to believe that patients' health status is not a main cause of the perception differences between male and female partners regarding patients' care dependency. Our conclusion is that more knowledge regarding these perception differences is needed. ${ }^{44}$ 
Although no differences were found in symptoms of depression between male and female patients, female partners rated patients more often with symptoms of depression compared to male partners. This could not be due to the symptoms of depression of partners themselves, because female partners showed an increased level of anxiety symptoms only. Janssen et a ${ }^{16}$ studied the agreement between patients with advanced chronic organ failure and their family caregivers regarding patients' symptoms. They concluded that family caregivers reported a higher number of symptoms than patients themselves and that patients' or family caregivers' gender were not determinants for clinically relevant differences. Nevertheless, research in partners' perceptions about patients' symptoms is very rare and should be expanded. ${ }^{44}$

The study at hand could not distinguish a difference between male and female partners' perceptions of the health status of patients with COPD. Additionally, male patients had a worse health status compared to female patients as assessed with the EQ-5D index score in patients themselves. A previous study concluded that proxies rated the patients as having a more impaired health status than the patients themselves. ${ }^{45}$ However, that study could not check for gender differences because their study population consisted solely of male patients with COPD.

\section{Clinical consequences}

Recently, the definition and ultimate goals of a collaborative self-management intervention in COPD patients were published. ${ }^{46}$ One of the goals referred to the patients' partner: "Establishing effective alliances with health care professionals, family, friends, and community." Therefore, all healthcare professionals who attempt to achieve self-management in patients with COPD should involve the patient's partner. Especially in case of female partners, attention should be paid to coping styles, symptoms of anxiety, and health status. We should also be aware of the social expectations of the idea that women act as "natural" caregivers. ${ }^{13}$ Because the feeling that caregiving is an assumed responsibility instead of a choice could affect the reaction to caregiving. ${ }^{13}$ For instance, when the social environment view the female caregiver as naturally better at caregiving, they might be less likely to perceive support as being needed, thus less likely to offer it.

So, obtaining an extensive overview of the patient-partner couple, including coping styles, health status, symptoms of anxiety, and caregiver burden, is necessary to be able to support the couple as affectively as possible. Future studies should explore more knowledge regarding gender-specific involvement of partners of patients with COPD.

\section{Methodological considerations}

Focus on the experience of mostly female caregivers was one of the most common limitations of studies included in the review by Grant et al. ${ }^{8}$ Therefore, a positive aspect of the current study is the fact that there is an almost equal amount of male and female patients and partners.

Nevertheless, some limitations of the present study should be acknowledged. First, the response rate was $42 \%$. So, unknown differences between participants and eligible patients or partners refusing participation may be present. Second, symptoms of anxiety and depression were assessed using the HADS, which is a validated and reliable questionnaire to screen for these symptoms. ${ }^{29}$ However, we did not use an instrument to diagnose an anxiety or depressive disorder according to the Fifth Edition of the Diagnostic and Statistical Manual of Mental Disorders. ${ }^{47}$ Lastly, we excluded non-partner and non-heterosexual relationships which may limit the generalizability of our findings.

\section{Conclusions and implications}

Consistent with our hypothesis, we found that female partners, compared to male partners, have more symptoms of anxiety, used different coping styles, and have a worse health status. In addition, perception differences between male and female partners did exist regarding patients' care dependency and patients' symptoms of depression. Yet, research in partners of patients with COPD is not very common and many research questions remain unanswered. Despite this, the current study showed that health care providers should pay attention to the needs of all partners of patients with COPD, but female partners in particular.

\section{Acknowledgments}

The authors are grateful to Mrs Jeannet Delbressine and Mrs Esther van den Bogaart for collection of the data and to Mrs Ivy Timmermans for input of the data.

The authors are grateful to the doctors and nurses of the following collaborating hospitals and their departments of respiratory medicine for their participation in this study: Zuyderland, Heerlen/Kerkrade/Brunssum, the Netherlands; Zuyderland, Sittard-Geleen, the Netherlands; Elkerliek Hospital, Helmond, the Netherlands; St Anna Hospital, Geldrop, the Netherlands.

\section{Author contributions}

All authors contributed toward data analysis, drafting and revising the paper and agree to be accountable for all aspects of the work. 


\section{Disclosure}

This project is financially supported by Lung Foundation Netherlands, Leusden, the Netherlands, Grant 3.4.12.024 and by a research grant from Boehringer-Ingelheim, the Netherlands. The authors report no conflicts of interest in this work.

\section{References}

1. From the global strategy for the diagnosis, management and prevention of COPD, global initiative for chronic obstructive lung disease (GOLD); 2016. Available from: http://www.goldcopd.org/. Accessed April 26, 2016.

2. Bischoff EW, Schermer TR, Bor H, Brown P, van Weel C, van den Bosch WJ. Trends in COPD prevalence and exacerbation rates in Dutch primary care. Br J Gen Pract. 2009;59(569):927-933.

3. Aryal S, Diaz-Guzman E, Mannino DM. COPD and gender differences: an update. Transl Res. 2013;162(4):208-218.

4. MartinezFJ, Curtis JL, Sciurba F, et al. Sex differences in severe pulmonary emphysema. Am J Respir Crit Care Med. 2007;176(3):243-252.

5. Ekstrom MP, Jogreus C, Strom KE. Comorbidity and sex-related differences in mortality in oxygen-dependent chronic obstructive pulmonary disease. PloS One. 2012;7(4):e35806.

6. Watson L, Schouten JP, Lofdahl CG, et al. Predictors of COPD symptoms: does the sex of the patient matter? Eur Respir J. 2006;28(2): 311-318.

7. Annegarn J, Meijer K, Passos VL, et al. Problematic activities of daily life are weakly associated with clinical characteristics in COPD. J Am Med Dir Assoc. 2012;13(3):284-290.

8. Grant M, Cavanagh A, Yorke J. The impact of caring for those with chronic obstructive pulmonary disease (COPD) on carers' psychological well-being: a narrative review. Int J Nurs Stud. 2012;49(11) 1459-1471.

9. Seamark DA, Blake SD, Seamark CJ, Halpin DM. Living with severe chronic obstructive pulmonary disease (COPD): perceptions of patients and their carers. An interpretative phenomenological analysis. Palliat Med. 2004;18(7):619-625.

10. Jacome C, Figueiredo D, Gabriel R, Cruz J, Marques A. Predicting anxiety and depression among family carers of people with chronic obstructive pulmonary disease. Int Psychogeriatr. 2014;26(7):1191-1199.

11. Navaie-Waliser M, Spriggs A, Feldman PH. Informal caregiving: differential experiences by gender. Med Care. 2002;40(12): 1249-1259.

12. Tuinstra J, Hagedoorn M, Van Sonderen E, et al. Psychological distress in couples dealing with colorectal cancer: gender and role differences and intracouple correspondence. Br J Health Psychol. 2004;9(Pt 4): 465-478.

13. Washington KT, Pike KC, Demiris G, Parker Oliver D, Albright DL, Lewis AM. Gender differences in caregiving at end of life: implications for hospice teams. J Palliat Med. 2015;18(12):1048-1053.

14. Hagedoorn M, Sanderman R, Ranchor AV, Brilman EI, Kempen GI, Ormel J. Chronic disease in elderly couples: are women more responsive to their spouses' health condition than men? J Psychosom Res. 2001;51(5):693-696.

15. Papastavrou E, Kalokerinou A, Papacostas SS, Tsangari H, Sourtzi P. Caring for a relative with dementia: family caregiver burden. $J A d v$ Nurs. 2007;58(5):446-457.

16. Janssen DJ, Spruit MA, Wouters EF, Schols JM. Symptom distress in advanced chronic organ failure: disagreement among patients and family caregivers. J Palliat Med. 2012;15(4):447-456.

17. Nakken N, Janssen DJ, van den Bogaart EH, Vercoulen JH, Wouters EF, Spruit MA. An observational, longitudinal study on the home environment of people with chronic obstructive pulmonary disease: the research protocol of the home sweet home study. BMJ Open. 2014;4(11):e006098.
18. Nakken N, Spruit MA, van den Bogaart EH, et al. Health status and morbidities in resident relatives of patients with COPD. J Am Med Dir Assoc. 2016;17(3):276.e1-278.e1.

19. Nakken N, Janssen DJA, van den Bogaart EHA, et al. Patient versus proxy-reported problematic activities of daily life in patients with COPD. Respirology. 2016. Epub 13.10.2016.

20. Smid DE, Wilke S, Jones PW, et al. Impact of cardiovascular comorbidities on COPD assessment test (CAT) and its responsiveness to pulmonary rehabilitation in patients with moderate to very severe COPD: protocol of the chance study. BMJ Open. 2015;5(7): $\mathrm{e} 007536$.

21. Vestbo J, Hurd SS, Agusti AG, et al. Global strategy for the diagnosis, management, and prevention of chronic obstructive pulmonary disease: GOLD executive summary. Am J Respir Crit Care Med. 2013; 187(4):347-365.

22. Rodriguez-Roisin R. Toward a consensus definition for COPD exacerbations. Chest. 2000;117(5 Suppl 2):398S-401S.

23. Carpenter CR, Bassett ER, Fischer GM, Shirshekan J, Galvin JE, Morris JC. Four sensitive screening tools to detect cognitive dysfunction in geriatric emergency department patients: brief Alzheimer's screen, short blessed test, Ottawa 3DY, and the caregiver-completed AD8. Acad Emerg Med. 2011;18(4):374-384.

24. Charlson ME, Pompei P, Ales KL, MacKenzie CR. A new method of classifying prognostic comorbidity in longitudinal studies: development and validation. J Chronic Dis. 1987;40(5):373-383.

25. Barelds DPH, Luteijn F, Arrindell WA. [Dutch Relationship Questionnaire]. Nederlandse relatievragenlijst (NRV): Handleiding. Pearson Assessment and Information B.V.: Amsterdam, The Netherlands; 2003.

26. Dijkstra A, Tiesinga LJ, Goossen WT, Dassen TW. Further psychometric testing of the Dutch Care Dependency Scale on two different patient groups. Int J Nurs Pract. 2002;8(6):305-314.

27. Dijkstra A, Tiesinga LJ, Plantinga L, Veltman G, Dassen TW. Diagnostic accuracy of the care dependency scale. J Adv Nurs. 2005; 50(4):410-416.

28. Schreurs PJG, Willige Gvd, Brosschot JF, Tellegen B, Graus GMH. [The Utrecht Coping List: UCL]. De Utrechtse Coping Lijst: UCL; Omgaan met problemen en gebeurtenissen. Amsterdam, The Netherlands: Pearson Assessment and Information B.V.; 1993. Dutch.

29. Zigmond AS, Snaith RP. The hospital anxiety and depression scale. Acta Psychiatr Scand. 1983;67(6):361-370.

30. Sherbourne CD, Stewart AL. The MOS social support survey. Soc Sci Med. 1991;32(6):705-714.

31. Cooper B, Kinsella GJ, Picton C. Development and initial validation of a family appraisal of caregiving questionnaire for palliative care Psychooncology. 2006;15(7):613-622.

32. Portney LD, Watkins MP. Foundations of Clinical Research: Applications to Practice. 3rd ed. NJ, USA: Upper Saddle River; 2008.

33. Lutzky SM, Knight BG. Explaining gender differences in caregiver distress: the roles of emotional attentiveness and coping styles. Psychol Aging. 1994;9(4):513-519.

34. Rose-Rego SK, Strauss ME, Smyth KA. Differences in the perceived well-being of wives and husbands caring for persons with Alzheimer's disease. Gerontologist. 1998;38(2):224-230.

35. Miller B, Cafasso L. Gender differences in caregiving: fact or artifact? Gerontologist. 1992;32(4):498-507.

36. Breeman S, Cotton S, Fielding S, Jones GT. Normative data for the Hospital Anxiety and Depression Scale. Qual Life Res. 2015;24(2):391-398.

37. Hooley PJ, Butler G, Howlett JG. The relationship of quality of life, depression, and caregiver burden in outpatients with congestive heart failure. Congest Heart Fail. 2005;11(6):303-310.

38. Tang ST, Chang WC, Chen JS, et al. Course and predictors of depressive symptoms among family caregivers of terminally ill cancer patients until their death. Psychooncology. 2013;22(6):1312-1318.

39. Cain CJ, Wicks MN. Caregiver attributes as correlates of burden in family caregivers coping with chronic obstructive pulmonary disease. J Fam Nurs. 2000;6(1):46-68. 
40. Limpawattana P, Theeranut A, Chindaprasirt J, Sawanyawisuth K, Pimporm J. Caregivers burden of older adults with chronic illnesses in the community: a cross-sectional study. J Community Health. 2013;38(1): $40-45$.

41. Lee KC, Chang WC, Chou WC, et al. Longitudinal changes and predictors of caregiving burden while providing end-of-life care for terminally ill cancer patients. J Palliat Med. 2013;16(6):632-637.

42. Lindqvist R, Carlsson M, Sjoden PO. Coping strategies and styles assessed by the Jalowiec Coping Scale in a random sample of the Swedish population. Scand J Caring Sci. 2000;14(3):147-154.

43. Stoilkova A, Wouters EF, Spruit MA, Franssen FM, Janssen DJ. The relationship between coping styles and clinical outcomes in patients with COPD entering pulmonary rehabilitation. COPD. 2013;10(3): 316-323.
44. Nakken N, Janssen DJ, van den Bogaart EH, et al. Informal caregivers of patients with COPD: home sweet home? Eur Respir Rev. 2015;24(137): 498-504.

45. Santiveri C, Espinalt M, Diaz Carrasco FX, Marin A, Miguel E, Jones PW. Evaluation of male COPD patients' health status by proxies. Respir Med. 2007;101(3):439-445.

46. Effing TW, Vercoulen JH, Bourbeau J, et al. Definition of a COPD self-management intervention: International Expert Group consensus. Eur Respir J. 2016;48(1):46-54.

47. American Psychiatric Association. Diagnostic and Statistical Manual of Mental Disorders. 5th ed. Arlington: American Psychiatric Association Publishing; 2013.

\section{Publish your work in this journal}

The International Journal of COPD is an international, peer-reviewed journal of therapeutics and pharmacology focusing on concise rapid reporting of clinical studies and reviews in COPD. Special focus is given to the pathophysiological processes underlying the disease, intervention programs, patient focused education, and self management protocols.

\section{Dovepress}

This journal is indexed on PubMed Central, MedLine and CAS. The manuscript management system is completely online and includes a very quick and fair peer-review system, which is all easy to use. Visit http://www.dovepress.com/testimonials.php to read real quotes from published authors.

Submit your manuscript here: http://www.dovepress.com/international-journal-of-chronic-obstructive-pulmonary-disease-journal 